\title{
Wide-field common-path incoherent correlation microscopy with a perfect overlapping of interfering beams
}

\author{
P. Bouchal \\ petr.bouchal@ceitec.vutbr.cz
}

\section{Z. Bouchal}

Central European Institute of Technology, Brno University of Technology, Technická 10, 61600 Brno, Czech Republic

Department of Optics, Palacký University, 17. listopadu 1192/12, 77146 Olomouc, Czech Republic

Incoherent correlation microscopy is recently discovered technique for digital imaging of three-dimensional objects in a quasimonochromatic spatially incoherent light. Its operation is based on wavefront division carried out by a spatial light modulator and capturing correlation recordings of the observed scene. To achieve image reconstruction, at least a partial overlapping of the signal and reference waves created by the spatial light modulator is necessary. In the known experimental configurations, the overlapping of interfering beams is strongly reduced in off-axis areas of the object and the image can be reconstructed only in a very small portion of the field of view provided by the used microscope objective lens. Here, we propose and successfully demonstrate modified experimental system working with two-component relay optics inserted between the microscope objective and the spatial light modulator and providing full overlapping of correlated beams in all areas of the field of view of the objective lens. The benefits and applicability of the proposed system design are clearly demonstrated on the imaging of the USAF resolution targets.

[DOI: http://dx.doi.org/10.2971/jeos.2013.13011]

Keywords: Correlation microscopy, digital holography, spatial light modulator, wide-field imaging, relay optics

\section{INTRODUCTION}

Digital holographic microscopy is a powerful imaging technique suitable for both metrology and bio-photonic applications including surface analysis or marker-free dynamic live cell imaging $[1,2]$. It is based on the principles of optical holography, which are advantageously combined with the recording of the holograms by a digital image sensor. The subsequent reconstruction of the holograms is performed numerically with a computer, and enables three-dimensional imaging without scanning. The digital holographic reconstruction was also used in the coherence-controlled microscope, where a quantitative phase contrast imaging and a coherence gating optical sectioning were demonstrated [3]. In recent years, the development of microscopy was favorably influenced by modern technologies enabling light shaping by a spatial light modulator (SLM). The SLM can be used in both illuminating and imaging paths of the optical microscope, where it ensures a structured specimen illumination, or amplitude and phase modulation of the spatial spectrum, respectively [4]. Using these phase modulation techniques, the spatial light interference microscopy (SLIM) [5, 6] and the spiral phase contrast imaging [7] were presented as powerful techniques capable of measuring nanoscale structures and dynamics in live cells or enhancing standard phase contrast methods. A variability of operations provided by the SLM was also used to design the universal microscope, that operatively combines the standard techniques of optical microscopy $[8,9]$. Light shaping by the SLM is also useful for digital microscopy. In the common-path phase-shifting lensless holographic microscopy, the SLM was used for a wave multiplexing ensuring a holographic recording and digital reconstruction of the specimen [10].
In its basic configuration, the digital holographic microscopy is limited to applications operating with coherent light needed to create holograms. Such a requirement is not necessary in the recently proposed Fresnel incoherent correlation holography that utilizes a SLM for holographic recording of observed objects illuminated by a quasi-monochromatic spatially incoherent light [11]. This technique removes many barriers for practical development of holography using incoherent light and is especially attractive for fluorescence microscopy. Recently, new modifications of the original system improving signal to noise ratio and allowing the use of sources with a broader spectrum have emerged $[12,13]$. The potential of the incoherent correlation microscopy has been further demonstrated by the imaging beyond the Rayleigh diffraction limit [14] and by the spiral recording and reconstruction of holograms resulting in selective edge contrast enhancement of three-dimensional objects [15]. The spiral edge contrast enhancement was successfully verified due to a hybrid coherence regime of the method leading to a fully coherent vortex reconstruction of the correlation records even though they were created in spatially incoherent light [16].

Although the incoherent correlation microscopy was presented in several experiments as a promising method with the wide application potential, the available field of view (FOV) of the method has not yet been investigated in any approach. In this paper, the FOV is studied in detail both theoretically and experimentally. In the investigated common-path incoherent correlation microscopy, the FOV is not directly determined by the microscope objective lens, but some restrictions 


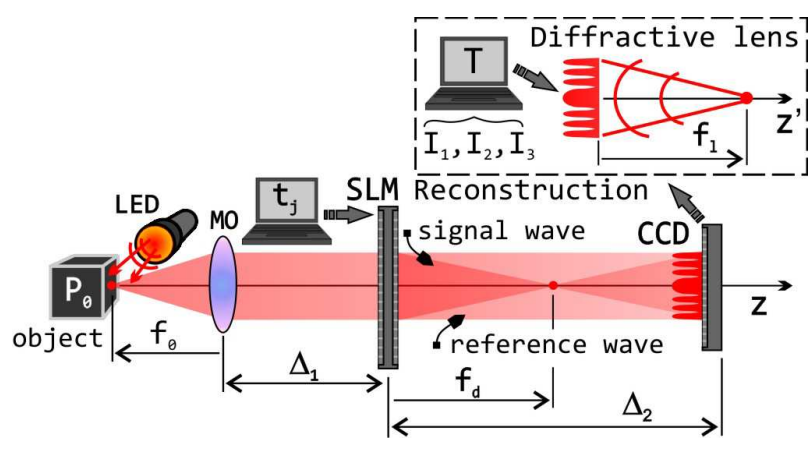

FIG. 1 Demonstration of the image reconstruction in the common-path incoherent correlation microscopy.

due to the correlation overlapping of interfering beams must be taken into account. Here we show that a reduction of the FOV caused by the insufficient correlation overlapping becomes particularly critical when using the microscope objective with a high magnification. The overlapping coefficient was defined by ray optics calculations and used to quantify the FOV in known experimental configurations. We also showed that a significant extension of the FOV is possible in the modified system using a two-component relay optics to achieve a perfect overlapping of the correlated beams. In the improved configuration, the common-path incoherent correlation microscopy allows image reconstruction in the full FOV of the used microscope objective. In addition, the full correlation overlapping improves image resolution, as mentioned in [14]. Theoretical predictions were successfully verified by the experiments demonstrating image reconstruction of the USAF resolution targets illuminated by a light-emitting diode.

\section{FOV IN BASIC CONFIGURATIONS OF THE COMMON-PATH INCOHERENT CORRELATION MICROSCOPY}

In the correlation microscopy, the FOV is not uniquely determined by geometric conditions for the passage of light as in the direct imaging, but a correlation overlapping of the interfering beams associated with the basic principle of the method must also be taken into consideration. A simplified scheme of the method is in Figure 1. To explain its operation, it is reasonable to assume that the observed object is composed of an infinite number of point sources that emit mutually uncorrelated light.

Light waves originating from separate object points are collimated by a microscope objective (MO) and directed towards the SLM. Each wave incident on the SLM is divided into two waves, called the signal and reference wave. These waves are spatially correlated because originate from the same object point. If their optical path difference does not exceed the coherence length of the used source, they can interfere. The interference pattern has a shape of the Fresnel zone plate and carries information about the amplitude and the threedimensional position of the relevant object point. A final record of the observed object is created by the addition of intensities of individual interference patterns because different points of the object are mutually uncorrelated. Information

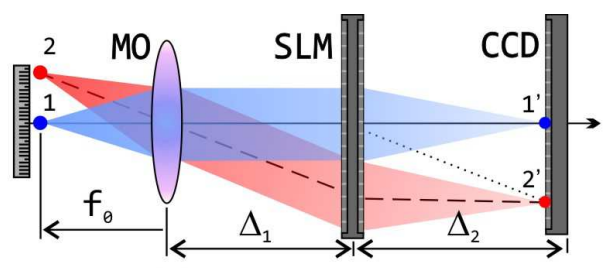

(a) direct imaging
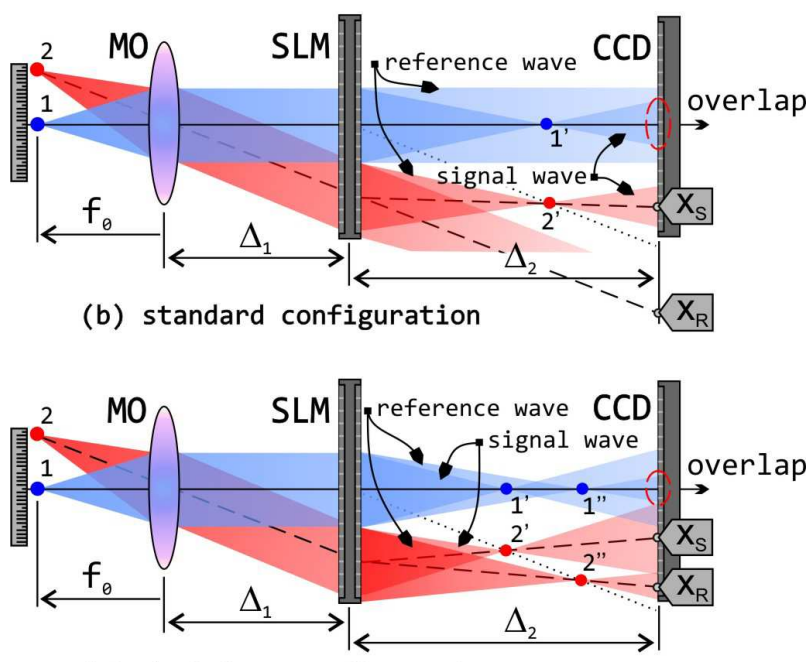

(c) dual lens configuration

FIG. 2 Illustration of ray trajectories in (a) the direct imaging and the common-path incoherent correlation microscopy with (b) standard and (c) dual lens configurations.

about the object is extracted by the processing and reconstruction of three object recordings acquired with different phase shifts of the signal wave.

Experimental setup shown in Figure 1 allows both direct and correlation imaging. In the direct imaging, the SLM produces a quadratic phase modulation and operates as the lens with a focal length $f_{d}$. The image on the CCD is then created by a two-lens system consisting of the MO and the SLM lens with the focal lengths $f_{0}$ and $f_{d}$, respectively. The FOV is determined by a ray tracing shown in Figure 2(a).

In the correlation imaging, each point of the object must be recorded as the Fresnel interference pattern. This is achieved in a common-path interferometer based on the wave splitting implemented by the SLM. Using the control software, the SLM transmission function $t_{j}=a \exp \left(i \theta_{r}\right)+b \exp \left(i \theta_{s}+i \vartheta_{j}\right)$ is set, where $a$ and $b$ are coefficients providing desired power distribution between the reference and signal waves, $\vartheta_{j}, j=1,2,3$, are constant phase shifts imposed on the signal wave, and $\theta_{r}$ and $\theta_{s}$ are phase functions for a wavefront transformation of the signal and reference wave. Three object recordings implemented with different phase shifts $\vartheta_{j}$ allow to eliminate the holographic twin image [17]. The phase functions $\theta_{r}$ and $\theta_{s}$ enable two different modes of imaging, here called the standard and dual lens imaging. In the standard common-path correlation imaging, $\theta_{r}$ corresponds to a constant phase shift. For object points located near the focal plane of the $\mathrm{MO}$, the reference wave corresponds to a collimated beam. The signal wave is generated by the quadratic phase function $\theta_{s}$ that represents a lens with the focal length $f_{d}$. In the dual lens imaging, both $\theta_{r}$ and $\theta_{s}$ are quadratic phase functions representing lenses with the focal lengths $f_{d_{1}}$ and $f_{d_{2}}$, respectively $[12,13]$. In this case, 
the correlation record of the object point is produced by interference of two spherical waves.

In the case of the correlation imaging, the FOV must be determined with respect to the desired overlapping of the signal and reference waves. The situation is illustrated in Figure 2(b) for the standard correlation imaging working with the plane reference wave and the spherical signal wave. Capturing the signal and reference waves on the CCD is not sufficient for imaging of the examined off-axis point. It can be successfully reconstructed only when the signal and reference waves are at least partially overlapped on the CCD. In the known configurations of the correlation imaging this condition can hardly be satisfied, especially if the best resolution is required. This is due to significant differences in the direction of propagation of the signal and reference waves. In the correlation imaging, the FOV is reduced in comparison with the direct imaging. This effect is remarkable when the MO with a high magnification is used.

Reduction of the FOV caused by the lack of correlation overlapping is estimated for both the standard and dual lens configurations using simple ray optics calculations.

\subsection{Standard configuration}

In the standard configuration, the SLM acts as a beam splitter, which transforms the signal wave as a lens with the focal length $f_{d}$, while the reference wave transmits unaffected. To estimate the degree of overlapping of interfering beams, the chief rays of the signal and reference waves must be traced and their positions on the CCD determined. The transverse coordinates of the intersections $x_{s}$ and $x_{r}$ are calculated by geometry shown in Figure 2(b) and can be written as

$$
\begin{aligned}
& x_{s}=\frac{x_{0}}{f_{0}} \Delta_{2}\left(1-\frac{\Delta_{1}}{f_{d}}+\frac{\Delta_{1}}{\Delta_{2}}\right), \\
& x_{r}=\frac{x_{0}}{f_{0}} \Delta_{2}\left(1+\frac{\Delta_{1}}{\Delta_{2}}\right),
\end{aligned}
$$

where $f_{0}$ is the focal length of the $\mathrm{MO}, \Delta_{1}$ is the separation distance between the MO and the SLM, $\Delta_{2}$ is a position of the CCD with respect to the SLM and $2 x_{0}$ is the FOV of the used $\mathrm{MO}$. The FOV of the $\mathrm{MO}$ is given by the field number $F$ and the magnification $m_{0}$,

$$
2 x_{0}=\frac{F}{m_{0}} .
$$

A separation of the signal and reference waves on the CCD can be conveniently defined by a distance of the spot centers and can be written as

$$
\Delta x=\left|x_{s}-x_{r}\right|=x_{0} \frac{\Delta_{1} \Delta_{2}}{f_{0} f_{d}} .
$$

The correlation overlapping also depends on the spot size of the detected signal and reference beams $D_{s}$ and $D_{r}$ given as

$$
D_{s}=\frac{\left|\Delta_{2}-f_{d}\right|}{f_{d}} D_{0}, \quad D_{r}=D_{0}
$$

where $D_{0}$ is the diameter of the exit pupil of the MO. It can be expressed by its numerical aperture $N A$ as $D_{0}=2 N A f_{0}$.
If we distinguish the beam spots according to their size as $D_{M}=\max \left\{D_{s}, D_{r}\right\}$ and $D_{m}=\min \left\{D_{s}, D_{r}\right\}$, a measure of the area overlapping of the interfering beams can be simply quantified by a coefficient $P$ defined as

$$
P=\left\{\begin{array}{rc}
1, & \text { for } \Delta x \leq D^{-} \\
\Delta S / S_{m}, & \text { for } D^{-}<\Delta x<D^{+} \\
0, & \text { for } \Delta x>D^{+}
\end{array}\right.
$$

where

$$
\begin{gathered}
\Delta S=\sum_{j=m, M} \Delta S_{j}, \quad S_{m}=\frac{\pi D_{m}^{2}}{4}, \\
\Delta S_{j}=\frac{D_{j}^{2}}{4} \arccos \left(1-\frac{2 h_{j}}{D_{j}}\right)-\left(\frac{D_{j}}{2}-h_{j}\right) \sqrt{h_{j}\left(D_{j}-h_{j}\right)}, \\
j=m, M, \\
h_{m}=\frac{\left(D^{+}-\Delta x\right)\left(\Delta x+D^{-}\right)}{2 \Delta x}, \quad h_{M}=\frac{\left(D^{+}-\Delta x\right)\left(\Delta x-D^{-}\right)}{2 \Delta x}, \\
D^{+}=\frac{D_{M}+D_{m}}{2}, \quad D^{-}=\frac{D_{M}-D_{m}}{2} .
\end{gathered}
$$

In this way, the coefficient $\mathrm{P}$ can be interpreted geometrically as the area of overlap of mutually shifted spots of interfering beams $\Delta S$. This area is normalized using smaller beam area $S_{m}=\min \left\{\pi D_{s}^{2} / 4, \pi D_{r}^{2} / 4\right\}$. A partial overlapping of beams is determined by $0<P<1$, whereas the limit value $P=1$ stands for a full overlapping of beams and $P=0$ for fully separated beams.

\subsection{Dual lens configuration}

In the dual lens configuration, the SLM splits an input wave into the signal and reference waves and transforms them as a dual lens with two different focal lengths $f_{d_{1}}$ and $f_{d_{2}}$, respectively. In this case, the signal and reference waves are spherical waves and the positions of their chief rays on the CCD are determined by

$$
\begin{aligned}
& x_{s}=\frac{x_{0}}{f_{0}} \Delta_{2}\left(1-\frac{\Delta_{1}}{f_{d 1}}+\frac{\Delta_{1}}{\Delta_{2}}\right), \\
& x_{r}=\frac{x_{0}}{f_{0}} \Delta_{2}\left(1-\frac{\Delta_{1}}{f_{d 2}}+\frac{\Delta_{1}}{\Delta_{2}}\right) .
\end{aligned}
$$

The geometrical separation of beam spots $\Delta x$ is obvious from Figure 2(c) and can be expressed as

$$
\Delta x=x_{0} \frac{\Delta_{1} \Delta_{2}}{f_{0}} \frac{\Delta f}{f_{d_{1}} f_{d_{2}}},
$$

where $\Delta_{f}=\left|f_{d_{1}}-f_{d_{2}}\right|$. The spot size of the signal and reference beams is given as

$$
D_{s}=\frac{\left|\Delta_{2}-f_{d_{1}}\right|}{f_{d_{1}}} D_{0}, \quad D_{r}=\frac{\left|\Delta_{2}-f_{d_{2}}\right|}{f_{d_{2}}} D_{0} .
$$

The overlapping coefficient $P$ can again be determined by Eq. (6) as a ratio of the overlapping area of the signal and reference beams $\Delta S$ and the beam spot area $S_{m}$. For object points close to the optical axis, the signal and reference waves have 


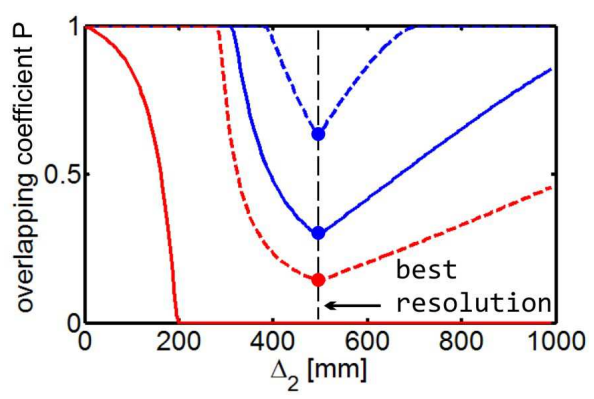

(a)

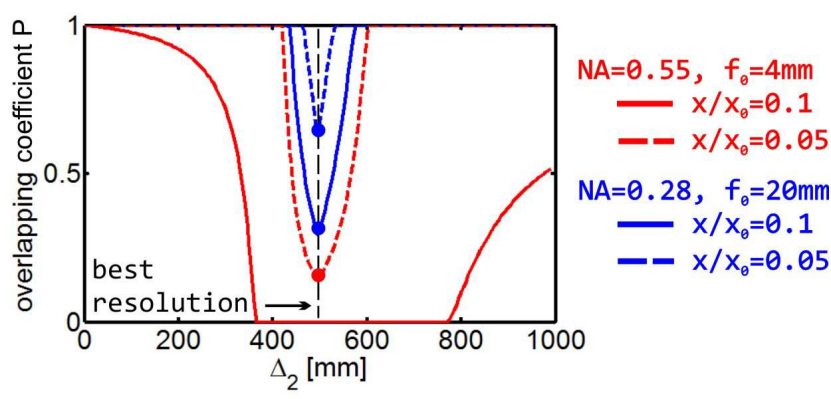

(b)

FIG. 3 Dependence of the correlation overlapping of interfering beams on the distance $\Delta_{2}$ for different parameters of the $\mathrm{MO}\left(\mathrm{NA}=0.28, f_{0}=20 \mathrm{~mm}\right.$ and $\mathrm{NA}=0.55, f_{0}=4$ $\mathrm{mm}$ ) and different parts of the relative FOV $\left(x / x_{0}=0.05\right.$ and $x / x_{0}=0.1$ ): (a) standard configuration with $\Delta_{1}=250 \mathrm{~mm}, f_{d}=247.5 \mathrm{~mm}$, (b) dual lens configuration with $\Delta_{1}=250 \mathrm{~mm}, f_{d_{1}}=400 \mathrm{~mm}$ and $f_{d_{2}}=649 \mathrm{~mm}$.

a full overlapping, $P=1$. For off-axis points, the overlapping is reduced and $P$ rapidly decreases with the distance $x$ of the object point from the axis. A smaller beam overlapping causes a quality degradation of the correlation imaging and the image reconstruction is impossible when the signal and reference beams are completely separated, $P=0$. The coefficient $P$ can thus be used to define the usable FOV of the correlation imaging. In numerical calculations, the relative FOV $x / x_{0}$ is used, where $2 x$ denotes the evaluated FOV in correlation microscopy and $2 x_{0}$ is the maximal FOV provided by the field number $F$ of the MO (in our analysis $F=26.5 \mathrm{~mm}$ is used). With both the standard and dual lens configurations, a required beam overlapping strongly limits the FOV and the correlation imaging utilizes only a fraction of the FOV available in a direct imaging provided by the MO. In Figure 3, the dependence of $P$ on $\Delta_{2}$ is illustrated for both the standard and dual lens configurations. The distance $\Delta_{2}$ determines the position of the CCD owing to the SLM and represents an important parameter of the correlation imaging. With the positioning of the CCD, the magnification of the correlation imaging can be changed. If the observed object is placed near the focal plane of the MO with the focal length $f_{0}$, the magnification is simply given by $m=\Delta_{2} / f_{0}$ [16]. The CCD position $\Delta_{2}$ also significantly affects the resolution of the correlation imaging. In the standard configuration, the best resolution is achieved with the condition $\Delta_{2}=2 f_{d}$, where $f_{d}$ is the focal length of the lens realized by the SLM. In the dual lens configuration, the best resolution is achieved in the setting $\Delta_{2}=2 f_{d_{1}} f_{d_{2}} /\left(f_{d_{1}}+f_{d_{2}}\right)$, where $f_{d_{1}}$ and $f_{d_{2}}$ are the focal lengths of the SLM lenses [13]. The correlation overlapping significantly changes with the CCD position $\Delta_{2}$ and reaches its minimum just in the most important positions, where the best resolution is achieved. This is clearly demonstrated for both standard Figure 3(a) and dual lens configuration Figure 3(b). At the plane of the best resolution, the coefficient $P$ has the same value in both configurations. For the $\mathrm{MO}$ with $N A=0.28$ and $f_{0}=20 \mathrm{~mm}$ its value is $P \approx 0.6$ for the relative FOV $x / x_{0}=0.05$ and $P \approx 0.3$ for $x / x_{0}=0.1$. For the $\mathrm{MO}$ with higher magnification ( $N A=0.55, f_{0}=4 \mathrm{~mm}$ ), the FOV is further reduced. For the relative FOV $x / x_{0}=0.05$, the coefficient $P \approx 0.15$ is obtained, while for $x / x_{0}=0.1$ the signal and reference beams are completely separated, $P=0$. If we assume with regard to the experimental experience that the overlapping $P \approx 0.3$ is sufficient for reconstructing the image with acceptable quality, the FOV for the correlation imaging is ap-

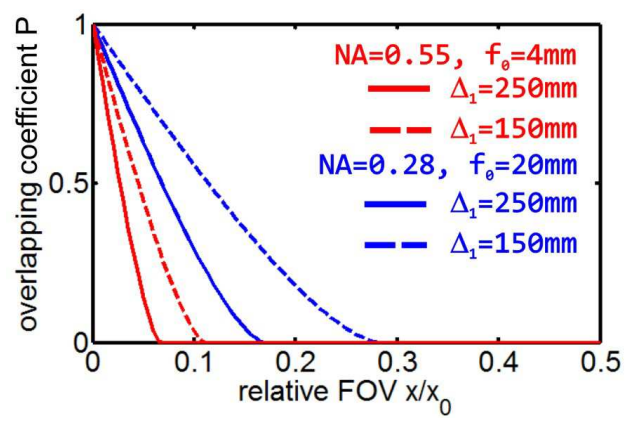

FIG. 4 Dependence of the correlation overlapping of interfering beams on the relative FOV for different parameters of the MO $\left(N A=0.28, f_{0}=20 \mathrm{~mm}\right.$ and $N A=0.55$, $f_{0}=4 \mathrm{~mm}$ ) and various settings $\Delta_{1}$.

proximately $10 \times$ smaller than in the direct imaging when the MO with $f_{0}=20 \mathrm{~mm}$ is used. In the case of the MO with $f_{0}=4 \mathrm{~mm}$, the correlation FOV is more than $20 \times$ smaller than in the direct imaging realized by the MO.

The size of the correlation FOV can be extended by shortening the distance $\Delta_{1}$ between the MO and the SLM. In Figure 4, the dependence of $P$ on the relative FOV is demonstrated for two different settings $\Delta_{1}=150$ and $250 \mathrm{~mm}$. The situation is identical for both standard and dual lens configurations and corresponds to the CCD positions $\Delta_{2}$ ensuring the best resolution. In terms of theory, $\Delta_{1}$ shortening leads to a steady overlapping improvement of the interfering beams and in the limit $\Delta_{1} \rightarrow 0$, the full overlapping is achieved. Unfortunately, the shortening of $\Delta_{1}$ has technical limitations. In most cases, a reflective SLM is used and a sufficient distance $\Delta_{1}$ is required for an insertion of a beam splitter and a polarizer or for an implementation of angular separation of incident and reflected beams. The distance $\Delta_{1}$ used in numerical simulation corresponds to real experimental possibilities.

\section{FOV IN THE COMMON-PATH INCOHERENT CORRELATION MICROSCOPY WITH RELAY OPTICS}

As was demonstrated, the overlapping of interfering beams is negatively influenced by increasing the distance between the MO and the SLM. As is clear from Eq. (4) and Eq. (9), an ideal beam overlapping is achieved in both standard and dual 


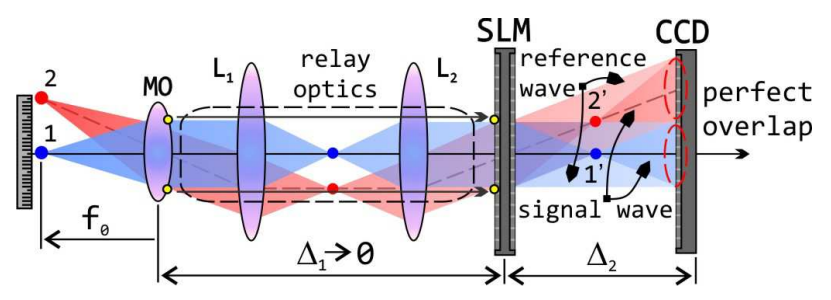

FIG. 5 Demonstration of a perfect correlation overlapping of interfering beams in the setup with two-component relay optics.

lens configurations when the SLM is placed just behind the objective lens, $\Delta_{1}=0$.

In this case, the chief rays of the signal and reference waves coincide so that $\Delta x=0$ for all points of the FOV of the used MO. Unfortunately, this condition cannot be met neither in the standard nor dual lens configurations for technical reasons. Here we present a novel setup modification where effects requiring the setting $\Delta_{1}=0$ are effectively achieved using twocomponent relay optics. An operation of the system is obvious from the ray paths illustrated in Figure 5. The setup is supplemented by two lens system that is placed between the $\mathrm{MO}$ and the SLM and ensures imaging of the exit pupil of the MO on an active area of the SLM. This is achieved when the inserted relay optics is configured as the $4 F$ system operating with the unitary magnification. The exit pupil of the $\mathrm{MO}$ is then placed at the front focal plane of the lens $L_{1}$ and the SLM is positioned at the back focal plane of the lens $L_{2}$. Such a configuration is adequate to the technically unattainable setting $\Delta_{1}=0$ in the standard and dual lens experiments. The relay optics ensures that the chief rays of the signal and reference waves coincide and a perfect overlapping of interfering beams is achieved in the full FOV of the used MO. This modification of the system is of great practical importance, because the FOV of the correlation imaging is as large as the FOV of the used MO regardless of the experimental parameters and the CCD positioning. The benefits of this method are evident from the experimental results.

The common-path correlation microscopy is essentially a digital holography method in which the continuous spatial distribution of optically generated holograms is sampled by the discrete sensitive pixels on a CCD array. In these systems, the space-bandwidth product (SBP) is introduced as a quantity that is directly related to the information capacity of the hologram [18]. To compare the demands imposed on detection devices, the SBP was determined for standard and dual lens configurations, and consequently also for both systems supplemented by the relay optics. In analysis, the SBP was traditionally defined as $S W=L_{x} W_{x}$, where $L_{x}$ is the transverse size of the detector needed to capture the hologram and $W_{x}$ stands for the highest spatial frequency contained in the hologram. In all examined configurations, $L_{x}=\max \left\{x_{s}, x_{r}\right\}$ was used, where $x_{s}$ and $x_{r}$ are the transverse positions of the spot centers of the signal and reference waves on the CCD. The maximal spatial frequency $W_{x}$ was determined by evaluating the relationship for a local frequency of the hologram. It can be written as $W_{x}=\left.(1 / 2 \pi)(\partial \Delta \Phi / \partial x)\right|_{x=x_{m}}$, where $\Delta \Phi$ is the phase difference of the signal and reference wave at the hologram plane and $x_{m}$ denotes the transverse coordinate where

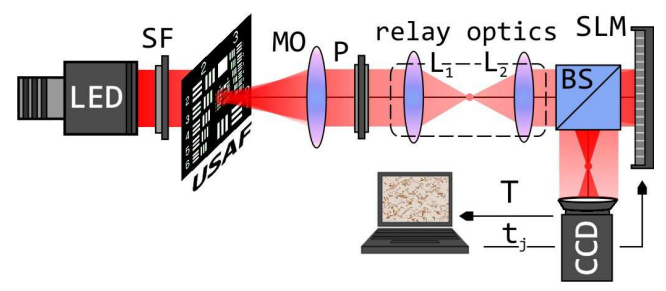

FIG. 6 Set-up for the common-path incoherent correlation microscopy using the relay optics for a perfect correlation overlapping and an extended FOV: SF - spectral filter, MO - microscope objective, P - polarizer, $L_{1}, L_{2}$ - two-component relay optics, BS beam splitter, SLM - spatial light modulator.

the local spatial frequency reaches its maximal value. In all examined configurations, the SBP was evaluated for the full FOV of the MO and the CCD positions for the best resolution. It was verified that the SBP becomes minimal in the dual lens configuration with the relay optics. In comparison with earlier experiments, the use of the relay system provides not only full correlation overlapping, but it also reduces the SPB at the same object size. In such a manner the relay optics reduces demands on the hologram detection.

\section{EXPERIMENTAL RESULTS}

Reduction of the FOV in standard and dual lens configurations and the proposed modification for the full overlapping of interfering beams were verified experimentally in the setup illustrated in Figure 6.

The collimated LED (Thorlabs, $625 \mathrm{~nm}$ ) with the spectral filter SF (FWHM $10 \mathrm{~nm}$ ) was used as an incoherent source of light. The FOV was examined using USAF resolution targets placed at the front focal plane of the Melles Griot $\mathrm{MO}(\mathrm{NA}=0.28$, $f_{0}=20 \mathrm{~mm}$ and $N A=0.55, f_{0}=4 \mathrm{~mm}$ ). The signal and reference waves were created using the SLM (Hamamatsu, $800 \times 600$ pixels) and the correlation records were captured on the CCD (Retiga $4000 \mathrm{R}$ ). The polarizer was used to achieve an optimal phase modulation by the SLM.

In initial experiments, the setup was used without relay optics to demonstrate a reduction of the FOV of the MO due to the lack of the correlation overlapping of the signal and reference waves. Subsequently, the experimental system was supplemented by the relay optics for a perfect overlapping of interfering beams. It was composed of two identical achromatic doublets with the focal length $150 \mathrm{~mm}$. As is evident from Figure 7, the correlation recordings made in the standard setup and the modified experiment with relay lenses are fundamentally different. When using the relay optics, the defocused correlated images of the USAF target created by the signal and reference waves are in a perfect overlapping and coincide (Figure 7(a)). In the standard configuration, a sufficient overlapping is reached only in the center of the FOV indicated by a dashed line in Figure 7(d). For off-axis parts of the object, the images created by the signal and reference waves are mutually shifted by a large distance $\Delta x$ and are almost separated. The correlation recordings can not be successfully reconstructed in this part of the FOV. In the dual lens imaging mode, the shift of interfering waves can be positively in- 


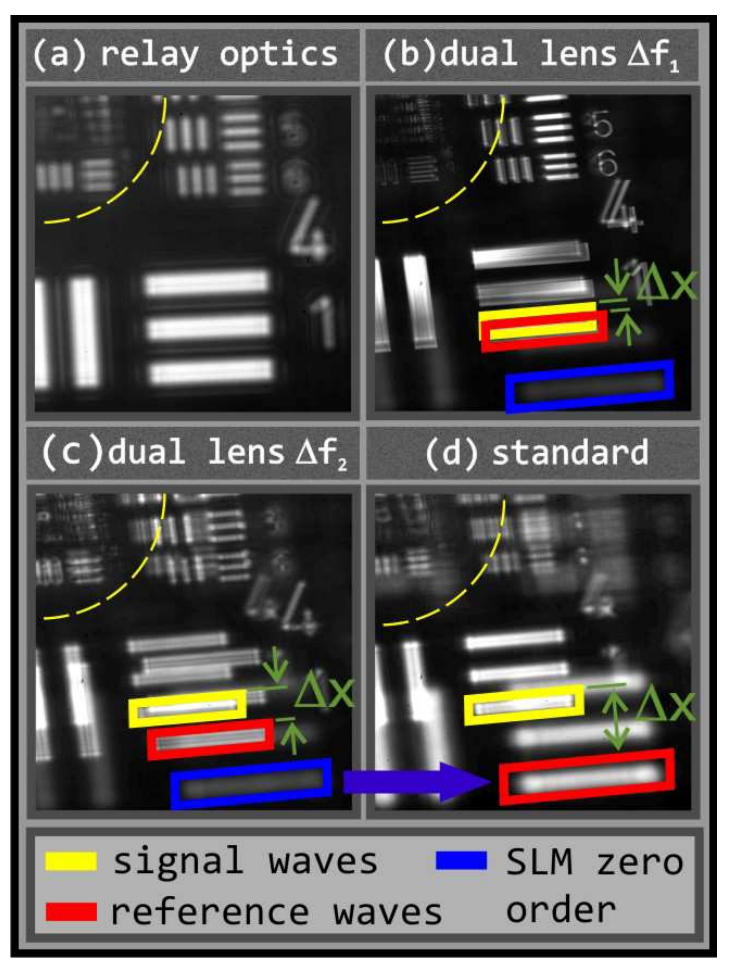

FIG. 7 Correlation recordings of the USAF resolution targets demonstrating various degrees of overlapping of the signal and reference waves in different experimental configurations: (a) perfect overlapping in the setup with relay optics, (b) dual lens configuration with $\Delta f_{1}=53 \mathrm{~mm}$ and (c) $\Delta f_{2}=249 \mathrm{~mm}$, (d) insufficient overlapping in standard configuration.

fluenced by reducing the distance $\Delta f$, which is the difference of the focal lengths of the SLM lenses. This effect is evident from Figures 7(b) and 7(c) illustrating the correlation recordings taken with $\Delta f_{1}=53 \mathrm{~mm}$ and $\Delta f_{2}=249 \mathrm{~mm}$, respectively. Unfortunately, the reduction of $\Delta f$ does not lead to an increase of the FOV, because the signal and reference waves reduce not only their lateral separation but also the areas of their spots and the overlapping area remains unchanged.

The correlation recordings shown in Figure 7 were taken by the Melles Griot MO with $N A=0.28$ and $f_{0}=20 \mathrm{~mm}$. The separation distance between the MO and the SLM was set to the minimal achievable value $\Delta_{1}=270 \mathrm{~mm}$. The best resolution of the system was ensured by the fixed position of the CCD, $\Delta_{2}=495 \mathrm{~mm}$, which was combined with the focal length of the SLM lens $f_{d}=247.5 \mathrm{~mm}$ in the standard configuration and $f_{d_{1}}=470 \mathrm{~mm}$ and $f_{d_{2}}=523 \mathrm{~mm}$ in the dual lens configuration.

The final images of the USAF resolution targets created by the processing and reconstruction of the demonstrated correlation recordings are shown in Figure 8. Advantages of the proposed modification of the setup are more apparent there, because the image is not reconstructed in areas with insufficient overlapping of interfering beams. The images of the groups 6 and 7 of the USAF target obtained by the reconstruction of the correlation recordings acquired in the standard and dual lens configurations are shown in Figures 8(a) and 8(c), respectively. The FOV specified by the correlation overlapping is approximately $2 x \approx 0.3 \mathrm{~mm}$ and remains the same in both the standard and dual lens configurations. This experimental finding

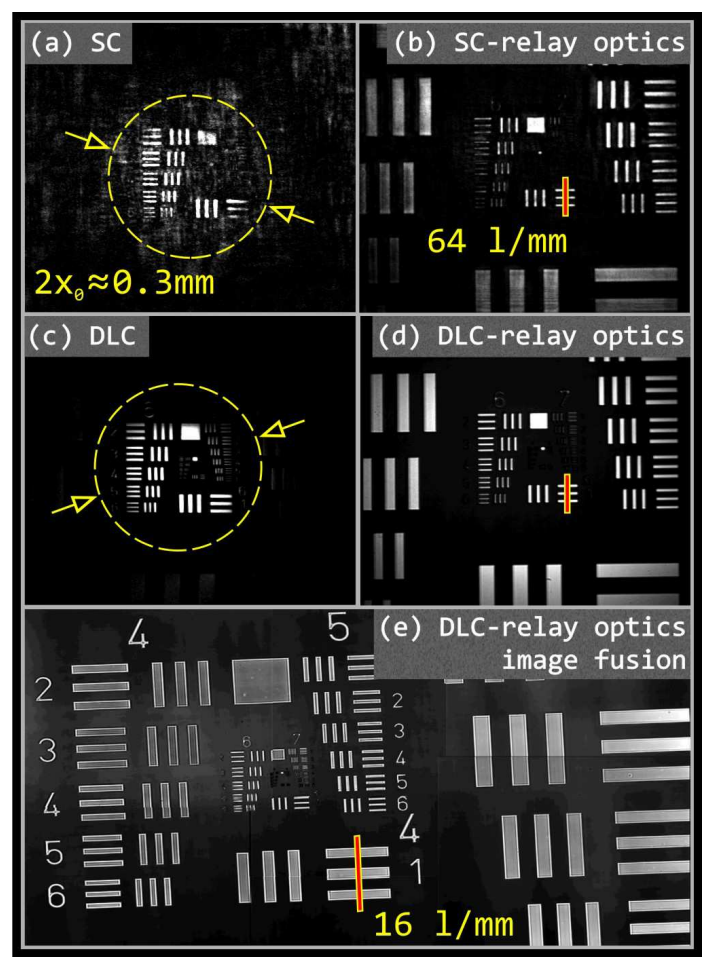

FIG. 8 Images of the USAF resolution target reconstructed from the correlation recordings captured in different experimental configurations using the Melles Griot MO with $N A=0.28$ and $f_{0}=20 \mathrm{~mm}$ : (a) standard configuration (SC), (b) SC with relay optics, (c) dual lens configuration (DLC), (d) DLC with relay optics, (e) DLC with relay optics and image reconstructed from the correlation recordings obtained by a fusion of nine partial CCD frames.

is consistent with the theoretical results demonstrated in Figure 3. Significant improvement of the FOV arises in both configurations when the relay lenses are used. The reconstructed images are shown in Figures 8(b) and 8(d). As is obvious, the FOV completely fills a detection area of the CCD in this case. Although the benefits of the relay system are indisputably demonstrated, the images 8(b) and 8(d) still cannot demonstrate the full potential of the modified setup, because the correlation field used in the image reconstruction is not fully captured by a single CCD frame. In order to demonstrate the full FOV when using the relay system, nine records were carried out at different lateral positions of the CCD while other system settings remained unchanged. The resulting correlation record used in the image reconstruction was then created by a fusion of the partial CCD records. The obtained wide-field image is shown in Figure 8(e). Compared with the image reconstructed from a single CCD frame, the groups 4 and 5 additionally may be seen there. Thus, the correlation FOV obtained in known configurations (Figures 8(a) and 8(c)) is only a fraction of the FOV available in the proposed system with the relay optics (Figure 8(e)).

To demonstrate a full functionality of the system modification in the conditions of digital microscopy, the experiment was also performed using the Melles Griot MO with higher magnification $\left(N A=0.55, f_{0}=4 \mathrm{~mm}\right)$. The results obtained are presented in Figure 9. Here, the finest elements of the groups 8 and 9 of the USAF target were reconstructed in the dual lens configuration that was adjusted to achieve the best resolution. Extension of the FOV in the system using the relay optics Fig- 


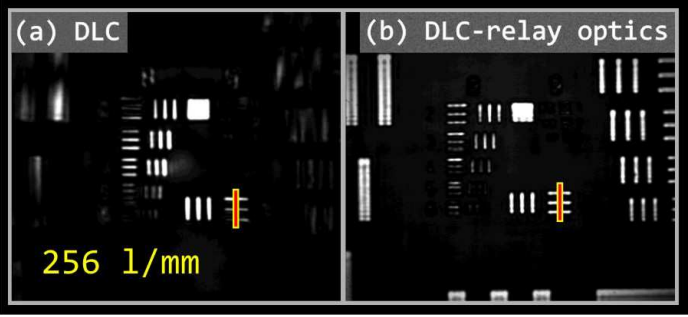

FIG. 9 Images of the USAF resolution target reconstructed from the correlation recordings captured in the dual lens configuration (DLC) using the Melles Griot MO with a higher magnification ( $N A=0.55$ and $f_{0}=4 \mathrm{~mm}$ ): (a) usual DLC and (b) DLC with the relay optics.

ure $9(b)$ is again significant in comparison with usual arrangement Figure 9(a). Moreover, the reconstructed image 9(b) still does not show all the available FOV, because the correlation field exceeded a detection area of the CCD and it was not fully captured by a single CCD frame.

\section{Conclusions}

In the paper, the basic configurations of incoherent correlation microscopy were examined in a connection with the available FOV. As the main result, a modification of the experimental setup has been proposed that provides a perfect overlapping of interfering beams. This design change is crucial because allows a significant expansion of the FOV and increases an effective numerical aperture resulting in the higher image resolution [14]. The partial results of the paper may be summarized as follows:

- It was first demonstrated that in the common-path incoherent correlation microscopy the image is reconstructed in the FOV determined by the conditions of the correlation overlapping of interfering beams.

- Ray optics calculations were performed allowing a simple estimation of the FOV by the conveniently defined overlapping coefficient.

- It was shown that the correlation FOV depends on the parameters of the experiment and significantly reduces the FOV of the used MO in both the standard and dual lens configurations. We verified that the reduction of the FOV caused by the lack of the correlation overlapping occurs mostly in the settings ensuring the best resolution and when the MO with higher magnification is used.

- The use of relay lenses was proposed to ensure a perfect correlation overlapping of interfering beams in all known configurations of the common-path correlation microscopy regardless of used parameters of the experiment.

The common-path incoherent correlation microscopy is a promising imaging technique supported by modern light shaping technologies. It allows numerical reconstruction of three-dimensional objects from the correlation recordings acquired in spatially incoherent quasi-monochromatic light.
Recently, the special properties of the method including sub-diffraction resolution [14], improved signal to noise ratio [12], extension of the permissible spectral bandwidth of the used light sources [13] or spiral edge enhancement [15] were successfully demonstrated and considered for application in fluorescence microscopy. The proposed modification of the setup resulting in a full correlation overlapping and extension of the FOV considerably increases optical parameters and application potential of the method.

\section{ACKNOWLEDGEMENT}

This work was supported by the Czech Ministry of Education, project No. MSM6198959213, the Technology Agency of the Czech Republic, project No. TE01020229, the IGA project of the Palacký University PrF 2012-05 and the project CEITEC - "Central European Institute of Technology" No. CZ.1.05/1.1.00/02.0068 from European Regional Development Fund. We thank Dr. J. Kapitán for stimulating discussions in initial stage of the research.

\section{References}

[1] P. Marquet, B. Rappaz, P. J. Magistretti, E. Cuche, Y. Emery, T. Colomb, and Ch. Depeursinge, "Digital holographic microscopy: a non invasive contrast imaging technique allowing quantitative visualization of living cells with sub wavelength accuracy," 0 pt. Lett., 30(5), 468-470 (2005).

[2] L. Lovicar, J. Komrska, and R. Chmelík, "Quantitative-phasecontrast imaging of a two-level surface described as a $2 \mathrm{D}$ linear filtering process," Opt. Express 18(20), 20585-20594 (2010)

[3] P. Kolman, and R. Chmelík, “Coherence-controlled holographic microscope," Opt. Express 18(21), 21990-22003 (2010).

[4] C. Maurer, A. Jesacher, S. Bernet, and M. Ritsch-Marte, "What spatial light modulators can do for optical microscopy," Laser Photonics Reviews 5, 81-101 (2011).

[5] Z. Wang, L. Millet, M. Mir, H. Ding, S. Unarunotai, J. Rogers, M. U. Gillette, and G. Popescu, "Spatial light interference microscopy (SLIM)," Opt. Express 19(2), 1016-1026 (2010).

[6] Z. Wang, I. S. Chun, X. Li, Z. Y. Ong, E. Pop, L. Millet, M. Gillette, and G. Popescu, "Topography and refractometry of nanostructures using spatial light interference microscopy," Opt. Lett. 35(2), 208-210 (2010).

[7] S. Furhapter, A. Jesacher, S. Bernet, M. Ritsch-Marte, "Spiral phase contrast imaging in microscopy," Opt. Express 13(3), 689-694 (2005).

[8] M. Warber, S. Zwick, T. Haist, and W. Osten, "SLM-based phasecontrast filtering for single and multiple image acquisition," P. Soc. Photo.-0pt. Ins. 7442, 74420E-74420E-12 (2009).

[9] M. Hasler, T. Haist, W. Osten, "SLM-based microscopy," P. Soc. Photo.-Opt. Ins. 8430, 84300V-84300V-8 (2012).

[10] V. Micó, and J. García, “Common-path phase-shifting lensless holographic microscopy," Opt. Lett. 35(23), 3919-3921 (2010).

[11] J. Rosen and G. Brooker, "Fluorescence incoherent color holography," Opt. Express 15(5), 2244-2250 (2007).

[12] X. Lai, Y. Zhao, X. Lv, Z. Zhou, and S. Zeng, "Fluorescence holography with improved signal-to-noise ratio by near image plane recording," Opt. Lett. 37(13), 2445-2447 (2012). 
[13] B. Katz, J. Rosen, R. Kelner, and G. Brooker, "Enhanced resolution and throughput of Fresnel incoherent correlation holography (FINCH) using dual diffractive lenses on a spatial light modulator (SLM)," Opt. Express 20(8), 9109-9121 (2012).

[14] J. Rosen, N. Siegel, and G. Brooker, "Theoretical and experimental demonstration of resolution beyond the Rayleigh limit by FINCH fluorescence microscopic imaging," Opt. Express 19(27), 26249-26268 (2011).

[15] P. Bouchal, and Z. Bouchal, "Selective edge enhancement in three-dimensional vortex imaging with incoherent light," Opt. Lett. 37(14), 2949-2951 (2012).
[16] P. Bouchal, J. Kapitán, R. Chmelík, and Z. Bouchal, "Point spread function and two-point resolution in Fresnel incoherent correlation holography," Opt. Express 19(16), 15603-15620 (2011).

[17] I. Yamaguchi and T. Zhang, "Phase-shifting digital holography," Opt. Lett. 22(16), 1268-1270 (1997).

[18] L. Xu, X. Peng, Z. Guo, J. Miao, and A. Asundi, "Imaging analysis of digital holography," Opt. Express 13(7), 2444-2452 (2005). 